\title{
Special issue: RNA biology in physiology and disease
}

\author{
Markus Kretz $^{1} \cdot$ Jan Medenbach $^{1} \cdot$ Gunter Meister $^{1}$
}

Received: 28 April 2016/Accepted: 28 April 2016/Published online: 14 May 2016

(C) Springer-Verlag Berlin Heidelberg 2016

Genetic information is stored as DNA in nuclear chromatin structures. For protein production, genes are transcribed to mRNA, which transports the information to ribosomes in the cytoplasm. This concept of 'information flow' is known for decades but with the advent of new sequencing technologies, it became rapidly apparent that RNA is not only coding but in fact the majority of RNA transcripts within a cell are non-coding. Such RNAs can have diverse functions including scaffolds for large RNA-protein assemblies (RNPs), catalytic activity (rRNA in the ribosome) or the regulation of coding and non-coding gene expression. Any of these non-coding RNAs have specific protein binding partners referred to as RNA-binding proteins (RBPs). For a long time, this extended protein class has not gained much attention. However, with the progress in understanding the function of various non-coding RNAs, these proteins move more and more into our focus. RBPs control the fate of their bound RNAs from synthesis to decay, governing all aspects of RNA biology and dynamically adjusting to cellular requirements. The finding that many RBPs harbor additional functions, e.g. enzymatic activity, has established a close link between RNA biology and in principle unrelated functions such as intermediary metabolism. In this special issue, review articles will address novel findings in RNA biology and discuss the role of RNA metabolism and its regulation in e.g. chronobiology. Furthermore, it will be highlighted in the individual reviews how mis-processing of RNA or mis-regulation of

Jan Medenbach

Jan.Medenbach@ur.de

1 University of Regensburg, Regensburg, Germany
RNA metabolism can change cellular homeostasis tilting the fine balance towards pathology and resulting in disease.

Non-coding RNAs such as tRNAs, rRNAs or small nuclear RNAs (snRNAs) are known for a long time and reasonably well understood. The discovery of microRNAs (miRNAs) and other small non-coding RNAs has boosted research into this direction. A plethora of studies has implicated small RNAs in literally all cellular pathways. MiRNAs play important developmental roles in many organisms but are in addition also vital for tissue generation and homeostasis. Furthermore, physiological processes such as insulin secretion and sugar metabolism, fat storage or the function of the cardiovascular system rely on the correct function of specific miRNAs. It is therefore not surprising that miRNAs have been associated with various diseases such as cardiovascular disorders, neurodegenerative disorders or cancer. Review articles within this special issue highlight the function of specific enzymes in miRNA production as well as the diverse sources of small RNAs in human cells. In addition, roles of miRNAs in the nervous system and in smooth muscle homeostasis are summarized.

In addition to multiple classes of short regulatory noncoding RNAs, the human genome encodes several thousand long non-protein coding transcripts $>200$ nucleotides in length. These long non-coding RNAs (lncRNAs) have emerged as key regulators of gene expression in many different cellular pathways and were frequently shown to act as part of protein-containing complexes. They can function as guides and scaffolds for proteins and were shown to act as structural decoys for transcription factors and other cellular molecules. LncRNAs have been implicated in a multitude of different diseases including many types of cancer and are crucial for development and 
homeostasis of a wide range of tissues. Several articles in this special issue will shed light on the roles of lncRNAs in control of tissue differentiation, regulation of metabolism and their involvement in disease development.

Analogous to epigenetics, the term epitranscriptomics has been coined, which accurately describes an extended layer of modulatory events that target a RNA transcript after synthesis.
To understand complex physiological pathways or diseases, a detailed understanding of gene regulation on the level of RNA is mandatory. This special issue combines three major epitranscriptomics directions: RBPs and RNA fate control, miRNA-guided gene silencing and the diverse functions of lncRNAs in gene regulation. 